\title{
Growth inhibition and enhanced chemosensitivity induced by down-regulation of Aurora-A in human renal cell carcinoma Caki-2 cells using short hairpin RNA
}

\author{
TOMOAKI TERAKAWA, HIDEAKI MIYAKE, MASAFUMI KUMANO and MASATO FUJISAWA \\ Division of Urology, Kobe University Graduate School of Medicine, Kobe 650-0017, Japan
}

Received November 30, 2010; Accepted April 8, 2011

DOI: $10.3892 / \mathrm{ol} .2011 .295$

\begin{abstract}
The objective of this study was to investigate the inhibitory effects of Aurora-A expression on the growth and chemosensitivity of Caki-2 cells in human renal cell carcinoma (RCC). Caki-2 cells were established, in which an expression vector containing short hairpin RNA (shRNA) targeting Aurora-A was introduced (Caki-2/sh-A). The growth and sensitivity of chemotherapeutic agents in Caki-2/sh-A cells were compared to those in Caki-2 cells transfected with control vector alone (Caki-2/C). The expression levels of both Aurora-A mRNA and protein in Caki-2/sh-A cells were less than $10 \%$ of those in Caki-2/C cells. The in vitro growth of Caki-2/sh-A cells was significantly inferior to that of Caki-2/C cells, and the proportion of Caki-2/sh-A cells in the G2-M phase was significantly greater compared to that of Caki-2/C cells. In addition, the expression level of Bax in Caki-2/sh-A cells was significantly higher as compared to that in Caki-2/C cells, while phosphorylated Akt in Caki-2/sh-A cells was markedly down-regulated compared to that in Caki-2/C cells. Among several chemotherapeutic agents examined, the most significant difference between Caki-2/sh-A and Caki-2/C cells was observed in the sensitivity to docetaxel. Thus, the $\mathrm{IC}_{50}$ value of docetaxel in Caki-2/sh-A cells was decreased by approximately $90 \%$ compared to that in Caki-2/C cells. Treatment of Caki-2/sh-A cells, but not Caki-2/C ones, with $5 \mathrm{nM}$ docetaxel resulted in the induction of apoptotic cell death accompanying the induction of p53. The findings suggest that the suppression of Aurora-A expression using shRNA is a useful therapeutic strategy against RCC through growth inhibition as well as enhanced chemosensitivity.
\end{abstract}

Correspondence to: Dr Hideaki Miyake, Division of Urology, Kobe University Graduate School of Medicine, 7-5-1 Kusunoki-cho, Chuo-ku, Kobe 650-0017, Japan

E-mail: hideakimiyake@hotmail.com

Key words: renal cell carcinoma, Aurora-A, growth, chemosensitivity

\section{Introduction}

Although equal segregation of chromosomes to the postmitotic daughter cells is ensured by the centrosome through the organization of the bipolar mitotic spindle during normal cell proliferation, multipolar mitotic spindles and a wide variety of centrosomal anomalies are frequently observed in cancer cells (1). The disruption of normal chromosomal segregation may be induced by such abnormalities, resulting in the production of aneuploid cells. The precise molecular mechanisms associated with the segregation of chromosomes have yet to be adequately characterized. However, a number of genes involved in regulating centrosome duplication have been cloned and intensively analyzed, including family members of mammalian Aurora homologues (2).

Aurora-A is a serine/threonine protein kinase that belongs to the Drosophila aurora and Saccharomyces cerevisiae Ip11 kinase family and has been shown to play an essential role in chromosome segregation and centrosome functions (3). Aurora-A has attracted special interest due to the localization on chromosome $20 \mathrm{q} 13$, a region frequently amplified in various types of human cancer specimens $(4,5)$. Furthermore, results of previous studies showed that the ectopic expression of Aurora-A in mouse NIH/3T3 cells and Rat 1 fibroblasts causes centrosome amplification and transformation in vitro as well as tumorigenesis in vivo $(6,7)$. Collectively, these findings suggested that, if overexpressed, Aurora-A plays a role as an oncogene through the abnormal regulation of centrosome function.

Renal cell carcinoma (RCC) is the most common malignancy of the adult kidney and annual estimates of newly diagnosed cases have been steadily increasing. RCC is characterized by various features that differ from those of other malignancies (8). In the field of RCC research, investigators showed the potential roles of centrosomal defects and chromosomal instability in disease progression (9). Previously, Aurora-A expression in RCC specimens, which was markedly elevated as compared to that found in normal kidney tissue, was found to be significantly associated with tumor grade and cell proliferative potential (10). Thus, clarifying the role of Aurora-A during the progression of RCC is crucial. In the present study, we analyzed the changes in the phenotypes of human RCC cells both in vitro and in vivo following the RNA interference-mediated knockdown of the Aurora-A protein. 


\section{Materials and methods}

Materials. Caki-2 cells derived from human RCC were purchased from the American Type Culture Collection (Rockville, MD, USA). Cells were maintained in RPMI (Life Technologies, Gaithersburg, MD, USA) supplemented with $10 \%$ heat-inactivated fetal bovine serum.

A chemically synthesized oligonucleotide encoding an Aurora-A short hairpin RNA (shRNA), including a loop motif, was inserted downstream of the U6 promoter of the pBAsi hU6 Pur DNA vector (Takara Bio, Otsu, Japan). The sequence of the shRNA targeted against Aurora-A was 5'-ATGCCCTGTCTTACTGTCA-3', corresponding to positions 853-871 within the Aurora-A mRNA sequence. Similarly, a control plasmid was constructed by randomizing the sequence of shRNA (5'-TCTTAATCGCGTATAAGGC-3').

Expression vectors were transfected into Caki-2 cells by liposome-mediated gene transfer methods as previously described (11). Briefly, either purified shRNA targeting Aurora-A cloned into the expression plasmid or control plasmid was added to Caki-2 cells after pre-incubation for 30 min with Lipofectamine (Invitrogen, San Diego, CA, USA) and serum-free OPTI-MEM (Life Technologies). Drug selection in $1.5 \mu \mathrm{g} / \mathrm{ml}$ puromycin (Sigma, St. Louis, MO, USA) started 3 days after transfection. Colonies were then harvested and expanded to cell lines.

Real-time RT-PCR. Total RNA $(1 \mu \mathrm{g})$ extracted from each cell line was reverse transcribed using an oligo $\mathrm{dT}$ and superscript pre-amplification system (Life Technologies). Real-time RT-PCR was performed using a sequence detector (Abi Prism 7700; PE Applied Biosystems, Foster City, CA, USA) based on the Taq Man assay according to the manufacturer's instructions, as previously described (12). The specimens were analyzed in triplicate and the mean values were used for quantification. The quantification value of Aurora-A mRNA was described as each value relative to $\beta$-actin mRNA.

Western blot analysis. Western blot analysis was performed as previously described (11). Briefly, samples containing equal amounts of protein $(15 \mu \mathrm{g})$ from the lysates of cultured cells were electrophoresed on an SDS-polyacrylamide gel and transferred to a nitrocellulose filter. The filters were blocked in phosphate-buffered saline (PBS) containing 5\% non-fat milk powder at $4^{\circ} \mathrm{C}$ overnight and then incubated for $1 \mathrm{~h}$ with antibodies against Aurora-A (TransGenic Inc., Kumamoto, Japan), total and phosphorylated Akt, PARP (Cell Signaling Technology, Danvers, MA, USA), p53 (Epitomics Inc., Burlingame, CA, USA) and $\beta$-actin (Santa Cruz Biotechnology, Santa Cruz, CA, USA). The filters were incubated for $30 \mathrm{~min}$ with horseradish peroxidase-conjugated secondary antibodies and specific proteins were then detected using an enhanced chemiluminescence Western blotting system (Amersham Pharmacia Biotech, Arlington Heights, IL, USA).

MTT assay. The inhibitory effects of chemotherapeutic agents on the in vitro growth of each cell line were assessed using MTT (Sigma) as previously described (11). Briefly, $1 \times 10^{4}$ cells were seeded in each well of 96-well microtiter plates and allowed to attach overnight. Cells were then treated with various concen- trations of docetaxel, cisplatin, etoposide or doxorubicin. After $48 \mathrm{~h}$ of incubation, $20 \mu \mathrm{l}$ of $5 \mathrm{mg} / \mathrm{ml}$ MTT in PBS was added to each well, followed by incubation for $4 \mathrm{~h}$ at $37^{\circ} \mathrm{C}$. Formazan crystals were dissolved in DMSO. The optical density was determined with a microculture plate reader (Becton Dickinson Labware, Lincoln Park, NJ, USA) at an absorbance of $540 \mathrm{~nm}$. Absorbance values were normalized to the values obtained for the vehicle-treated cells to determine the percentage of surviving cells. Each assay was performed in triplicate.

Flow cytometric analysis and DNA degradation assay. The cell cycle phase distribution was assessed by a flow cytometric analysis of DNA content. Cells were fixed in $70 \%$ ethanol, and incubated with $1 \mu \mathrm{g} / \mathrm{ml}$ RNaseA for $30 \mathrm{~min}$. Following centrifugation, the cells were resuspended in $1 \mathrm{ml}$ of $50 \mu \mathrm{g} / \mathrm{ml}$ propidium iodide (Sigma) and incubated for 30 min. DNA content was evaluated using a FACScan flow cytometer and quantified using CellQuest software (Becton Dickinson). Each experiment was performed in triplicate.

Nucleosomal DNA degradation was quantified by a Cell Death Detection ELISA kit using antihistone antibody (Roche, Mannheim, Germany) as previously described (13). Briefly, $1 \times 10^{5}$ cells were seeded in 5-cm culture dishes and allowed to adhere overnight. Following treatment with docetaxel under the same schedule as described above, cells were harvested and assays were performed in triplicate according to the manufacturer's instructions.

Statistical analysis. Differences between the two groups were compared using the unpaired t-test. Statistical calculations were performed using Statview 5.0 software (Abacus Concepts, Inc., Berkley, CA, USA). P $<0.05$ was considered to be statistically significant.

\section{Results}

Cell lines.Caki-2cells were transfected with an expression vector containing the shRNA targeted against Aurora-A or vector alone. After drug selection, a number of stable independent clones were established and two of these clones (Caki-2/sh-A\#1 and Caki-2/sh-A\#2), in which the vector containing Aurora-A shRNA had been introduced, and control vector-transfected Caki-2 cells (Caki-2/C) were selected for further studies.

Expression levels of Aurora-A in Caki-2 sublines. Real-time RT-PCR and Western blotting were subsequently performed to evaluate the expression levels of Aurora-A mRNA and protein, respectively, in Caki-2/C, Caki-2/sh-A\#1 and Caki-2/ sh-A\#2 cells. As shown in Fig. 1, abundant levels of Aurora-A expression were observed in Caki-2/C cells at both the mRNA and protein levels, whereas Aurora-A expression levels in Caki-2/sh-A\#1 and Caki-2/sh-A\#2 cells were inhibited to $<10 \%$ compared to those in Caki-2/C cells.

Effect of Aurrora-A suppression on the growth of Caki-2 sublines. The MTT assay was performed to compare the growth patterns of the Caki-2 sublines. As shown in Fig. 2A, the growth of Caki-2/sh-A\#1 and Caki-2/sh-A\#2 cells was significantly inhibited compared to that of $\mathrm{Caki}-2 / \mathrm{C}$ cells, showing growth inhibition by $\sim 40 \%$ on day 5 . We then 


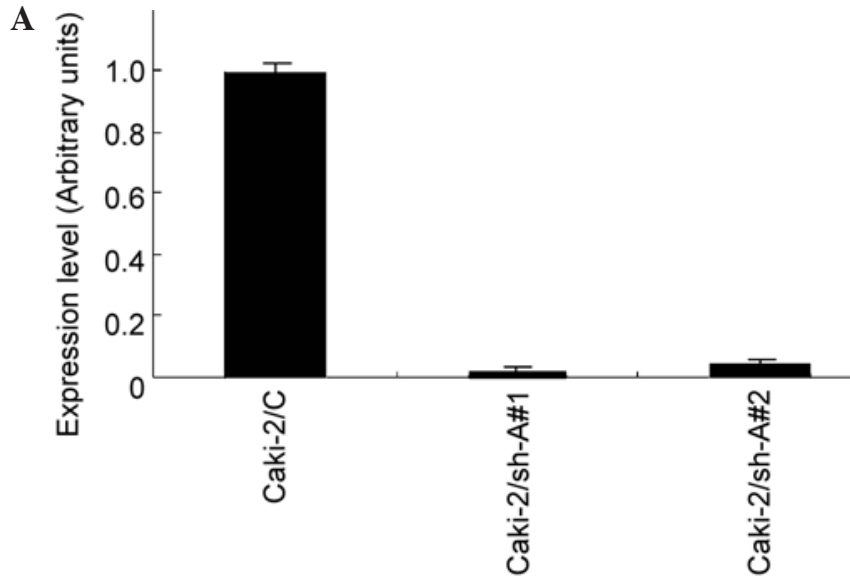

B

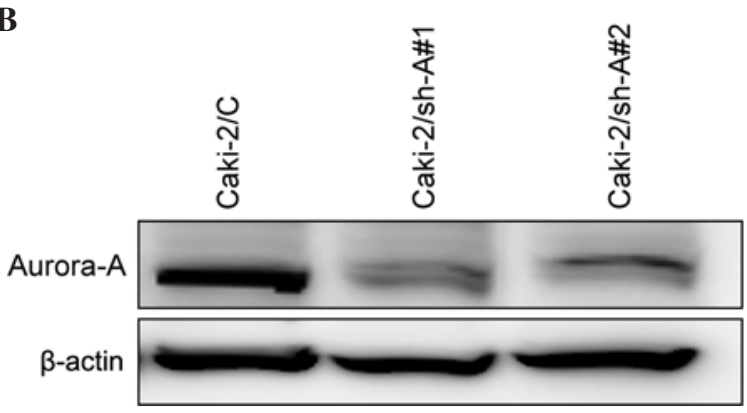

Figure 1. Expression levels of Aurora-A in Caki-2 sublines (Caki-2/C, control vector-only transfected cell line; Caki-2/sh-A\#1 and Caki-2/sh-A\#2, Aurora-A short hairpin RNA-transfected cell lines). (A) Real-time RT-PCR and (B) Western blotting were performed in order to analyze the expression levels of Aurora-A mRNA and protein, respectively, in each cell line.

investigated the cell cycle distributions in Caki-2 cell sublines by flow cytometry. The proportions of Caki-2/sh-A\#1 and Caki-2/sh-A\#2 cells in the G2-M phase were significantly greater than those of Caki-2/C cells (Fig. 2B).

Western blot analysis was used to determine whether the decrease in Aurora-A production by Caki- 2 cells modulates the expression levels of various molecules involved in signal transduction pathways as well as apoptosis. Of these molecules, the expression levels of phosphorylated Akt in Caki-2/sh-A\#1 and Caki-2/sh-A\#2 cells were markedly down-regulated compared to those in Caki-2/C cells, while the Bax expression levels in Caki-2/sh-A\#1 and Caki-2/sh-A\#2 cells were significantly higher than those in Caki-2/C cells (Fig. 2C).

To determine whether the inhibition of Aurora-A expression results in enhanced chemosensitivity in RCC, the Caki-2 sublines were treated with various doses of chemotherapeutic agents, such as docetaxel, cisplatin, etoposide and doxorubicin . Despite the lack of significant differences in sensitivities to cisplatin and doxorubicin in the Caki-2 sublines, Caki-2/sh-A\#1 and Caki-2/sh-A\#2 cells were more sensitive to docetaxel and etoposide than Caki-2/C cells. In other words, the $\mathrm{IC}_{50}$ value of docetaxel and that of etoposide in both Caki-2/sh-A\#1 and Caki-2/sh-A\#2 cells were reduced by $\sim 90$ and $50 \%$, respectively, as compared to those in Caki-2/C cells (data not shown). Accordingly, docetaxel, which was shown to have the most powerful cytotoxic effect on Caki-2/sh-A\#1 and Caki-2/ sh-A\#2 cells, was used in the subsequent study associated with chemosensitivity.

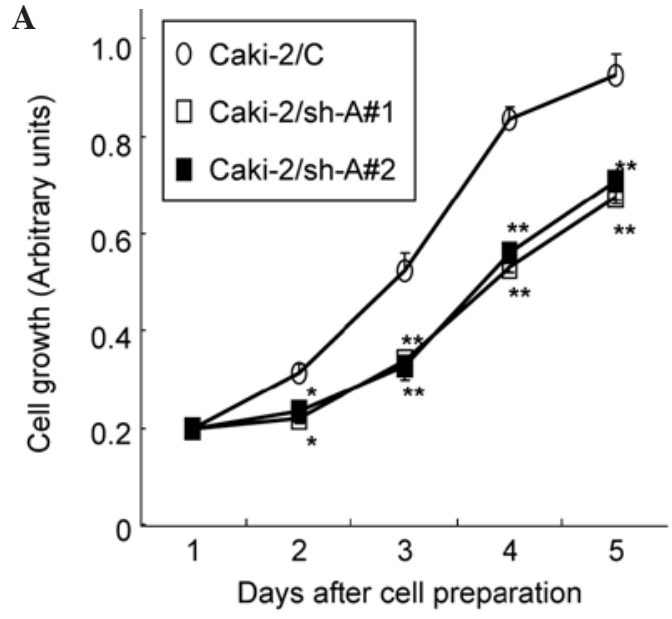

B
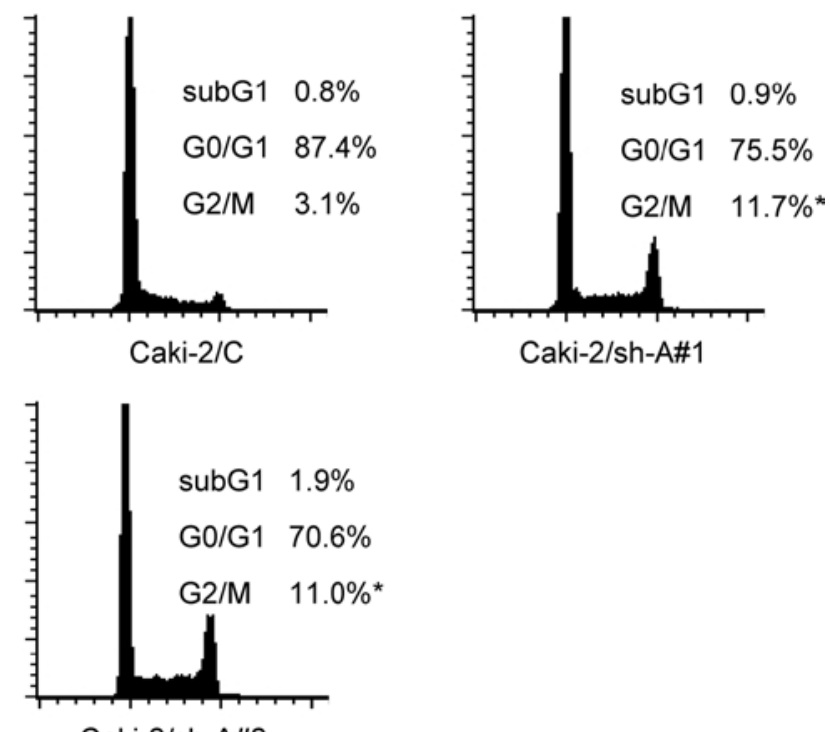

Caki-2/sh-A\#2

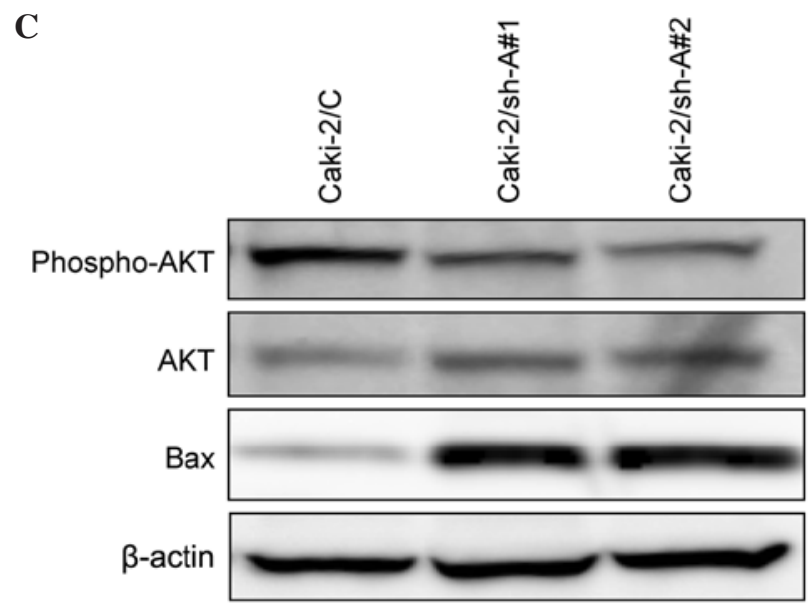

Figure 2. Effect of Aurora-A suppression on the growth of Caki-2 sublines. (A) In vitro proliferation of Caki-2 sublines $(\mathrm{Caki}-2 / \mathrm{C}$, control vector-only transfected cell line; Caki-2/sh-A\#1 and Caki-2/sh-A\#2, Aurora-A short hairpin RNA-transfected cell lines) was measured daily in triplicate by MTT assay. Bars, SD. ${ }^{* *}$ and ${ }^{*}$, significantly different from Caki-2/C $(\mathrm{p}<0.01$ and $\mathrm{p}<0.05$, respectively). (B) Cell cycle distribution of the Caki-2 sublines was evaluated by flow cytometry. Proportion of cells in the sub-G1, G0-G1 and G2-M phase are indicated. "Significantly different from Caki-2/C $(\mathrm{p}<0.05)$. (C) Proteins were extracted from the Caki-2 sublines cultured in standard medium. Both phosphorylated and total Akt and Bax protein levels were analyzed by Western blotting. 
A

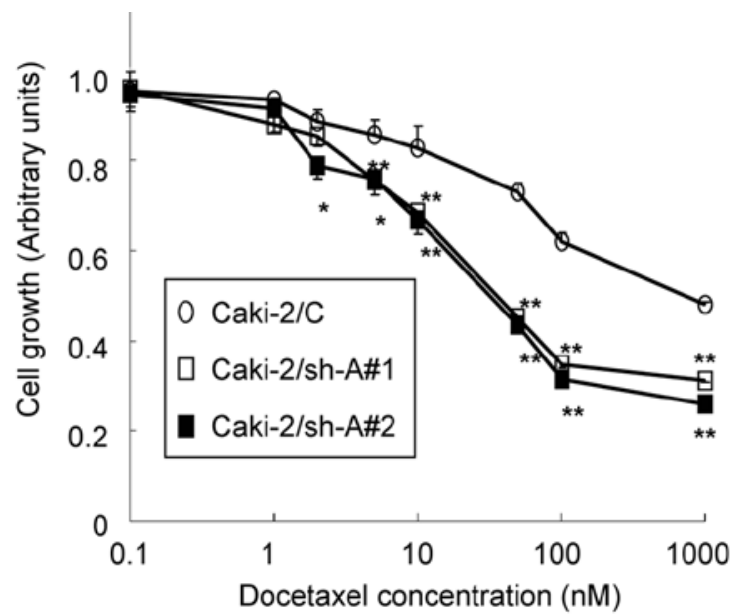

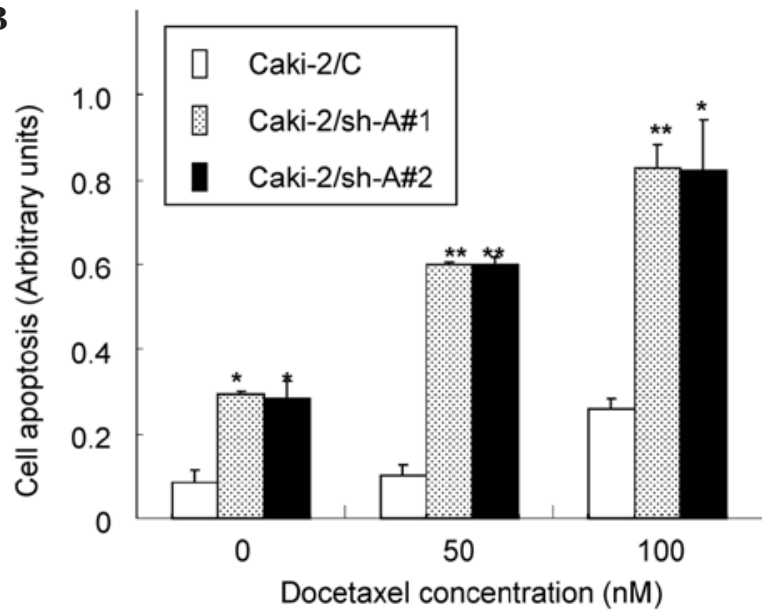

C

\begin{tabular}{|c|c|c|c|c|c|c|c|c|c|}
\hline \multicolumn{3}{|c|}{ Caki-2/C } & \multicolumn{3}{|c|}{ Caki-2/sh-A\#1 } & \multicolumn{3}{|c|}{ Caki-2/sh-A\#2 } & \multirow{3}{*}{$\begin{array}{l}\text { Docetaxel } \\
\text { concentration } \\
(\mathrm{nM})\end{array}$} \\
\hline 0 & 5 & 10 & 0 & 5 & 10 & 0 & 5 & 10 & \\
\hline re & 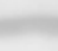 & 5 & $n$ & $=$ & $=$ & e & $=$ & $m$ & \\
\hline
\end{tabular}

Cleaved-PARP

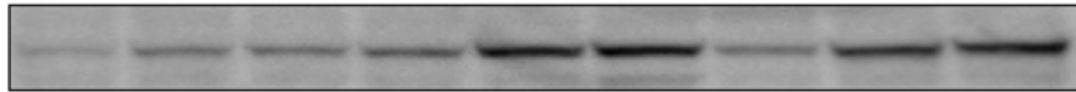

Total PARP

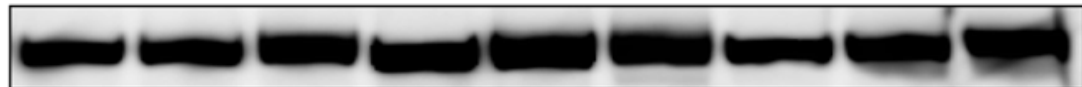

$\beta$-actin

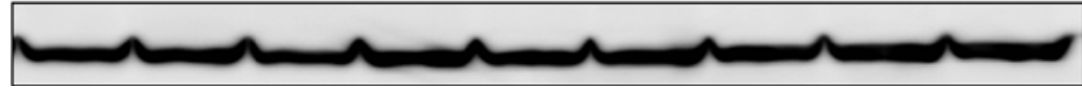

Figure 3. Effect of Aurora-A suppression on the sensitivity of Caki-2 sublines to docetaxel. (A) Caki-2 sublines (Caki-2/C, control vector-only transfected cell line; Caki-2/sh-A\#1 and Caki-2/sh-A\#2, Aurora-A short hairpin RNA-transfected cell lines) were treated with the indicated doses of docetaxel. After 48 h of incubation, cell growth was determined in triplicate by MTT assay. Bars, SD. ${ }^{* *}$ and ${ }^{*}$, significantly different from control (p<0.01 and p<0.05, respectively). (B) Caki-2 sublines were treated with the indicated doses of docetaxel. After $48 \mathrm{~h}$ of incubation, cell apoptosis was determined in triplicate cultures by an ELISA kit using antihistone antibody. Bars, SD. ${ }^{* *}$ and ${ }^{*}$, significantly different from control $(\mathrm{p}<0.01$ and $\mathrm{p}<0.05$, respectively). (C) Proteins were extracted from the Caki-2 sublines treated with 5 and $10 \mathrm{nM}$ of docetaxel for $48 \mathrm{~h}$. Then, p53 and both total and cleaved PARP protein levels were analyzed by Western blotting.

Effect of Aurora-A suppression on the sensitivity of Caki-2 sublines to docetaxel. As shown in Fig. 3A, docetaxel showed dose-dependent cytotoxicity in all Caki-2 sublines. However, the growth of Caki-2/sh-A\#1 and Caki-2/sh-A\#2 cells was significantly inhibited by docetaxel relative to the Caki-2/C cells in a dose-dependent manner. Furthermore, apoptotic changes in the Caki-2 sublines following treatment with various concentrations of docetaxel were investigated. The proportions of cells undergoing apoptotic cell death in Caki-2/ sh-A\#1 and Caki-2sh-A\#2 cells were significantly greater compared to those in Caki-2/C cells (Fig. 3B). In addition, treatment of Caki-2/sh-A\#1 and Caki-2sh-A\#2 cells with docetaxel resulted in the induction of p53 and the cleavage of PARP, but not of Caki-2/C cells (Fig. 3C).

\section{Discussion}

Considering the frequent observation of chromosomal abnormalities in human cancer cells, malignant disease progression may be associated with changes in the phenotype induced by genes involved in chromosomal segregation (1). Among such genes, Aurora-A has attracted great interest, since various studies have shown that the overexpression of Aurora-A induces disruption of normal cell cycle progression, resulting in the promotion of oncogenic transformation $(2,3,6,7)$. Furthermore, the up-regulation of Aurora-A expression and its significant correlation to conventional prognostic factors in a variety of human malignancies has been demonstrated $(3,14,15)$. We also previously reported that the expression of Aurora-A in RCC tissue is significantly stronger than that in normal kidney tissue, and that the Aurora-A expression level in RCC appears to be significantly correlated to tumor grade and cell proliferative activity (10). However, the role of Aurora-A during the progression of RCC has yet to be determined. Subsequently, this study investigated the effect of suppressed Aurora-A expression in human RCC Caki-2 cells on changes in their growth and chemosensitivity.

Initially, Caki-2 sublines were established in which Aurora-A expression was decreased by approximately $90 \%$ as compared to that in control Caki-2 cells by introducing an expression plasmid containing shRNA targeted against Aurora-A. Using the Caki-2 sublines, it has subsequently been shown that the suppression of Aurora-A causes a significant accumulation of cells in the G2-M phase, resulting in the 
marked inhibition of Caki-2 cell proliferation. These findings suggest that Aurora-A is an essential molecule for regulating the growth of RCC cells. Thus, Aurora-A may be a suitable molecular target for the inhibition of cell proliferation in RCC.

Of note is the mechanism involved in Aurora-A-mediated growth regulation in Caki-2 sublines. Of several molecules implicated in major signal transduction pathways, Akt pathway appeared to be markedly inactivated by the down-regulation of Aurora-A in Caki-2 cells. We showed a significant induction of Bax protein in Caki-2 cells by suppressing the expression of Aurora-A. These findings have been supported by various previous studies showing activation of the Akt pathway characterized by the exertion of an anti-apoptotic impact on various types of cancer cells $(16,17)$. In their study, Cha et al reported treatment with histone deacetylase inhibitor resulting in the induction of G2-M arrest and apoptosis through the degradation of Aurora-A (18).

Changes in chemosensitivity following the down-regulation of Aurora-A expression in Caki-2 cells were then investigated. Of several chemotherapeutic agents examined in this study, docetaxel has shown the most potent cytotoxic effect on Aurora-A shRNA-transfected Caki- 2 cells by reducing the $\mathrm{IC}_{50}$ value by approximately $90 \%$. Furthermore, the degree of chemosensitivity to docetaxel in the Caki-2 sublines is closely proportional to that of cells undergoing apoptosis, accompanying the induction of p53 and the cleavage of PARP. Taxanes, including docetaxel, have been shown to stabilize microtubules by binding tubulin and to interfere with microtubule disassembly. In other words, these agents are capable of stopping cell cycle progression, causing cells in the $\mathrm{M}$ phase to accumulate at the metaphase-anaphase transition and subsequently promote apoptosis (19). Taken together, these findings indicate that the synergistic induction of apoptosis in cancer cells may be achieved by the suppression of Aurora-A expression combined with the use of docetaxel. Similarly, the enhancement of docetaxel-induced apoptosis by the inhibition of Aurora-A expression using a variety of cancer cell lines, such as head and neck, esophageal and breast cancer cell lines has also been reported (20-22).

In conclusion, the suppression of Aurora-A expression by human RCC Caki-2 cells using shRNA inhibits their growth through the modulation of signal transduction and apoptotic pathways. Additionally, a synergistic cytotoxic effect is achieved in Caki-2 cells by combining the suppression of Aurora-A expression with docetaxel treatment. Collectively, these findings suggest that targeting Aurora-A using RNAinterfering technology in combination with docetaxel is a novel promising strategy for the treatment of patients with advanced RCC.

\section{References}

1. Nigg EA: Mitotic kinases as regulators of cell division and its checkpoints. Nat Rev Mol Cell Biol 2: 21-32, 2001.

2. Carmena $M$ and Earnshaw WC: The cellular geography of aurora kinase. Nat Rev Mol Cell Biol 4: 842-854, 2003.

3. Warner SL, Bearss DJ, Han H and von Hoff DD: Targeting aurora-2 kinase in cancer. Mol Cancer Ther 2: 589-595, 2003.

4. Fukushige S, Waldman FM, Kimura M, Abe T, Furukawa T, Sunamura M, Kobari M and Horii A: Frequent gain of copy number on the long arm of chromosome 20 in human pancreatic adenocarcinoma. Genes Chromosomes Cancer 19: 161-169, 1997.
5. Isola JJ, Kallioniemi OP, Chu LW, Fuqua SA, Hilsenbeck SG, Osborne CK and Waldman FM: Genetic aberrations detected by comparative genomic hybridization predict outcome in nodenegative breast cancer. Am J Pathol 147: 905-911, 1995.

6. Zhou H, Kuang J, Zhong L, Kuo WL, Gray JW, Sahin A, Brinkley BR and Sen S: Tumor amplification kinase STK15/ BTAK induces centrosome amplification, aneuploidy and transformation. Nat Genet 20: 189-193, 1998.

7. Bischoff JR, Anderson L, Zhu Y, Mossie K, Ng L, Souza B, Schryver B, Flanagan P, Clairvoyant F, Ginther C, Chan CS, Novotny M, Slamon DJ and Plowman GD: A homologue of Drosophila aurora kinase is oncogenic and amplifies in human colorectal cancers. EMBO J 17: 3052-3065, 1998.

8. Drucker BJ: Renal cell carcinoma: current status and future prospects. Cancer Treat Rev 31: 536-545, 2005.

9. Bodmer D, van den Hurk W, van Groningen JJ, Eleveld MJ, Martens GJ, Weterman MA and van Kessel AG: Understanding familial and non-familial renal cell cancer. Hum Mol Genet 11: 2489-2498, 2002.

10. Kurahashi T, Miyake H, Hara I and Fujisawa M: Significance of Aurora-A expression in renal cell carcinoma. Urol Oncol 25: 128-133, 2007.

11. Miyake H, Nelson C, Rennie PS and Gleave ME: Overexpression of insulin-like growth factor binding protein-5 helps accelerate progression to androgen-independence in the human prostate LNCaP tumor model through activation of phosphatidylinositol 3'-kinase pathway. Endocrinology 141: 2257-2265, 2000.

12. Miyake H, Hara I, Kurahashi T, Inoue TA, Eto H and Fujisawa M: Quantitative detection of micrometastases in pelvic lymph nodes in patients with clinically localized prostate cancer by real-time reverse transcriptase-PCR. Clin Cancer Res 13: 1192-1197, 2007.

13. Miyake H, Yamanaka K, Muramaki M, Hara I and Gleave ME: Therapeutic efficacy of adenoviral-mediated p53 gene transfer is synergistically enhanced by combined use of antisense oligodeoxynucleotide targeting clusterin gene in a human bladder cancer model. Neoplasia 7: 171-179, 2005.

14. Tanaka E, Hashimoto Y, Ito T, Okumura T, Kan T, Watanabe G, Imamura M, Inazawa J and Shimada Y: The clinical significance of Aurora-A/STK15/BTAK expression in human esophageal squamous cell carcinoma. Clin Cancer Res 11: 1827-1834, 2005.

15. Gritsko TM, Coppola D, Paciga JE, Yang L, Sun M, Shelley SA, Fiorica JV, Nicosia SV and Cheng JQ: Activation and overexpression of centrosome kinase BTAK/Aurora-A in human ovarian cancer. Clin Cancer Res 9: 1420-1426, 2003.

16. Guan Z, Wang XR, Zhu XF, Huang XF, Xu J, Wang LH, Wan XB, Long ZJ, Liu JN, Feng GK, Huang W, Zeng YX, Chen FJ and Liu Q: Aurora-A, a negative prognostic marker, increases migration and decreases radiosensitivity in cancer cells. Cancer Res 67: 10436-10444, 2007.

17. Liu X, Shi Y, Woods KW, Hessler P, Kroeger P, Wilsbacher J, Wang J, Wang JY, Li C, Li Q, Rosenberg SH, Giranda VL and Luo Y: Akt inhibitor a-443654 interferes with mitotic progression by regulating aurora a kinase expression. Neoplasia 10: 828-837, 2008.

18. Cha TL, Chuang MJ, Wu ST, Sun GH, Chang SY, Yu DS, Huang SM, Huan SK, Cheng TC, Chen TT, Fan PL and Hsiao PW: Dual degradation of aurora A and B kinases by the histone deacetylase inhibitor LBH589 induces G2-M arrest and apoptosis of renal cancer cells. Clin Cancer Res 15: 840-850, 2009.

19. Zhou J and Giannakakou P: Targeting microtubules for cancer chemotherapy. Current Med Chem 5: 665-671, 2005.

20. Mazumdar A, Henderson YC, El-Naggar AK, Sen S and Clayman GL: Aurora kinase A inhibition and paclitaxel as targeted combination therapy for head and neck squamous cell carcinoma. Head Neck 31: 625-634, 2009

21. Tanaka E, Hashimoto Y, Ito T, Kondo K, Higashiyama M, Tsunoda S, Ortiz C, Sakai Y, Inazawa J and Shimada Y: The suppression of aurora-A/STK15/BTAK expression enhances chemosensitivity to docetaxel in human esophageal squamous cell carcinoma. Clin Cancer Res 13: 1331-1340, 2007.

22. Lee HH, Zhu Y, Govindasamy KM and Gopalan G: Downregulation of Aurora-A overrides estrogen-mediated growth and chemoresistance in breast cancer cells. Endocr Relat Cancer 15: 765-775, 2008. 